\title{
Sharp bounds for Toader mean in terms of arithmetic, quadratic, and Neuman means
}

\author{
Jun-Feng Li ${ }^{1}$, Wei-Mao Qian² and Yu-Ming Chu ${ }^{1 *}$
}

"Correspondence:

chuyuming2005@126.com

1 School of Mathematics and

Computation Sciences, Hunan City

University, Yiyang, 413000, China

Full list of author information is

available at the end of the article

\begin{abstract}
In this paper, we present the best possible parameters $\alpha, \beta \in \mathbb{R}$ and $\lambda, \mu \in(1 / 2,1)$ such that the double inequalities

$\alpha N_{A Q}(a, b)+(1-\alpha) A(a, b)<T^{*}(a, b)<\beta N_{A Q}(a, b)+(1-\beta) A(a, b)$,

$\mathrm{Q}[\lambda a+(1-\lambda) b, \lambda b+(1-\lambda) a]<T^{*}(a, b)<\mathrm{Q}[\mu a+(1-\mu) b, \mu b+(1-\mu) a]$ hold for all $a, b>0$ with $a \neq b$, where $T^{*}(a, b), A(a, b), Q(a, b)$ and $N_{Q A}(a, b)$ are the Toader, arithmetic, quadratic, and Neuman means of $a$ and $b$, respectively.
\end{abstract}

MSC: $26 \mathrm{E} 60$

Keywords: Toader mean; arithmetic mean; quadratic mean; Neuman mean

\section{Introduction}

For $a, b>0$ the Toader mean $T^{*}(a, b)[1]$ is given by

$$
T^{*}(a, b)=\frac{2}{\pi} \int_{0}^{\pi / 2} \sqrt{a^{2} \cos ^{2} \theta+b^{2} \sin ^{2} \theta} d \theta .
$$

It is well known that the Toader mean satisfies

$$
T^{*}(a, b)=R_{E}\left(a^{2}, b^{2}\right)
$$

for all $a, b>0$, where

$$
R_{E}(a, b)=\frac{1}{\pi} \int_{0}^{\infty} \frac{[a(t+b)+b(t+a)] t}{(t+a)^{3 / 2}(t+b)^{3 / 2}} d t
$$

stands for the symmetric complete elliptic integral of the second kind (see [2-4]), therefore it cannot be expressed in terms of the elementary transcendental functions.

Recently, the Toader mean $T^{*}(a, b)$ has been the subject of intensive research. In particular, many remarkable inequalities for the Toader mean can be found in the literature [5-12].

Let $p \in \mathbb{R}$ and $a, b>0$. Then the $p$ th power mean $M_{p}(a, b)$ is defined by

$$
M_{p}(a, b)=\left(\frac{a^{p}+b^{p}}{2}\right)^{1 / p} \quad(p \neq 0), \quad M_{0}(a, b)=\sqrt{a b}
$$

(c) $2015 \mathrm{Li}$ et al. This article is distributed under the terms of the Creative Commons Attribution 4.0 International License (http://creativecommons.org/licenses/by/4.0/), which permits unrestricted use, distribution, and reproduction in any medium, provided you give appropriate credit to the original author(s) and the source, provide a link to the Creative Commons license, and indicate if changes were made. 
It is well known that $M_{p}(a, b)$ is continuous and strictly increasing with respect to $p \in \mathbb{R}$ for fixed $a, b>0$ with $a \neq b$.

Vuorinen [13] conjectured that the inequality

$$
M_{3 / 2}(a, b)<T^{*}(a, b)
$$

holds for all $a, b>0$ with $a \neq b$. This conjecture was proved by Qiu and Shen [14], and Barnard et al. [15], respectively.

Alzer and Qiu [16] presented a best possible upper power mean bound for the Toader mean as follows:

$$
T^{*}(a, b)<M_{\log 2 /(\log \pi-\log 2)}(a, b)
$$

for all $a, b>0$ with $a \neq b$.

Chu et al. [17] proved that the inequality

$$
T^{*}(a, b)<T(a, b)
$$

holds for all $a, b>0$ with $a \neq b$, where $T(a, b)=(a-b) /[2 \arctan ((a-b) /(a+b))]$ is the second Seiffert mean.

Another important mean of two positive real numbers $a$ and $b$ is the Schwab-Borchardt mean $[18,19]$

$$
S B(a, b)= \begin{cases}\frac{\sqrt{b^{2}-a^{2}}}{\arccos (a / b)}, & a<b, \\ \frac{\sqrt{a^{2}-b^{2}}}{\cosh ^{-1}(a / b)}, & a>b, \\ a, & a=b,\end{cases}
$$

where $\cosh ^{-1}(x)=\log \left(x+\sqrt{x^{2}-1}\right)$ is the inverse hyperbolic cosine function.

It is well known that the Schwab-Borchardt mean $S B(a, b)$ is strictly increasing in both $a$ and $b$, nonsymmetric and homogeneous of degree 1 . Many symmetric bivariate means are special cases of the Schwab-Borchardt mean. For example, $P(a, b)=(a-$ $b) /[2 \arcsin ((a-b) /(a+b))]=S B[G(a, b), A(a, b)]$ is the first Seiffert mean, $T(a, b)=(a-$ b) $/[2 \arctan ((a-b) /(a+b))]=S B[A(a, b), Q(a, b)]$ is the second Seiffert mean, $M(a, b)=(a-$ $b) /\left[2 \sinh ^{-1}((a-b) /(a+b))\right]=S B[Q(a, b), A(a, b)]$ is the Neuman-Sándor mean, $L(a, b)=$ $(a-b) /\left[2 \tanh ^{-1}((a-b) /(a+b))\right]=S B[A(a, b), G(a, b)]$ is the logarithmic mean, where $G(a, b)=\sqrt{a b}, A(a, b)=(a+b) / 2$ and $Q(a, b)=\sqrt{\left(a^{2}+b^{2}\right) / 2}$ are the geometric, arithmetic, and quadratic means of $a$ and $b$, respectively.

Very recently, Neuman [20] introduced the Neuman mean,

$$
N(a, b)=\frac{1}{2}\left[a+\frac{b^{2}}{S B(a, b)}\right]
$$

and presented the explicit formula for $N_{A Q}(a, b) \equiv N[A(a, b), Q(a, b)]$ as follows:

$$
N_{A Q}(a, b)=\frac{1}{2} A(a, b)\left[1+\left(1+v^{2}\right) \frac{\arctan (v)}{v}\right]
$$

and proved that the double inequality 


$$
T(a, b)<N_{A Q}(a, b)<Q(a, b)
$$

holds for all $a, b>0$ with $a \neq b$, where $v=(a-b) /(a+b)$.

Inequalities (1.2), (1.3), and (1.5) lead to

$$
A(a, b)=M_{1}(a, b)<M_{3 / 2}(a, b)<T^{*}(a, b)<N_{A Q}(a, b)<Q(a, b)
$$

for all $a, b>0$ with $a \neq b$.

Let $a, b>0$ with $a \neq b$ be fixed and $f(x)=Q[x a+(1-x) b, x b+(1-x) a]$. Then it is not difficult to verify that $f(x)$ is continuous and strictly increasing on $[1 / 2,1]$. Note that

$$
f\left(\frac{1}{2}\right)=A(a, b)<T^{*}(a, b)<Q(a, b)=f(1) .
$$

Motivated by inequalities (1.6) and (1.7), it is natural to ask: what are the best possible parameters $\alpha, \beta \in \mathbb{R}$ and $\lambda, \mu \in(1 / 2,1)$ such that the double inequalities

$$
\begin{aligned}
& \alpha N_{A Q}(a, b)+(1-\alpha) A(a, b)<T^{*}(a, b)<\beta N_{A Q}(a, b)+(1-\beta) A(a, b), \\
& Q[\lambda a+(1-\lambda) b, \lambda b+(1-\lambda) a]<T^{*}(a, b)<Q[\mu a+(1-\mu) b, \mu b+(1-\mu) a]
\end{aligned}
$$

hold for all $a, b>0$ with $a \neq b$ ? The main purpose of this paper is to answer this question.

\section{Lemmas}

In order to prove our main results we need several lemmas, which we present in this section.

For $r \in(0,1)$ the complete elliptic integrals $\mathcal{K}(r)$ and $\mathcal{E}(r)$ of the first and second kinds are defined by

$$
\mathcal{K}(r)=\int_{0}^{\pi / 2}\left(1-r^{2} \sin ^{2} t\right)^{-1 / 2} d t
$$

and

$$
\mathcal{E}(r)=\int_{0}^{\pi / 2}\left(1-r^{2} \sin ^{2} t\right)^{1 / 2} d t
$$

respectively. We clearly see that

$$
\mathcal{K}\left(0^{+}\right)=\mathcal{E}\left(0^{+}\right)=\frac{\pi}{2}, \quad \mathcal{K}\left(1^{-}\right)=\infty, \quad \mathcal{E}\left(1^{-}\right)=1
$$

and the Toader mean $T^{*}(a, b)$ given by (1.1) can be expressed as

$$
T^{*}(a, b)= \begin{cases}2 a \mathcal{E}\left(\sqrt{1-(b / a)^{2}}\right) / \pi, & a>b, \\ 2 b \mathcal{E}\left(\sqrt{1-(a / b)^{2}}\right) / \pi, & a<b \\ a, & a=b\end{cases}
$$

$\mathcal{K}(r)$ and $\mathcal{E}(r)$ satisfy the formulas (see [21], Appendix E, p.474,475)

$$
\frac{d \mathcal{K}(r)}{d r}=\frac{\mathcal{E}(r)-\left(1-r^{2}\right) \mathcal{K}(r)}{r\left(1-r^{2}\right)}, \quad \frac{d \mathcal{E}(r)}{d r}=\frac{\mathcal{E}(r)-\mathcal{K}(r)}{r},
$$




$$
\mathcal{E}\left(\frac{2 \sqrt{r}}{1+r}\right)=\frac{2 \mathcal{E}(r)-\left(1-r^{2}\right) \mathcal{K}(r)}{1+r}
$$

Lemma 2.1 (See [21], Theorem 1.25) Let $-\infty<a<b<\infty, f, g:[a, b] \rightarrow(-\infty, \infty)$ be continuous on $[a, b]$ and differentiable on $(a, b)$, and $g^{\prime}(x) \neq 0$ on $(a, b)$. Iff $(x) / g^{\prime}(x)$ is increasing (decreasing) on $(a, b)$, then so are

$$
\frac{f(x)-f(a)}{g(x)-g(a)}, \quad \frac{f(x)-f(b)}{g(x)-g(b)}
$$

If $f^{\prime}(x) / g^{\prime}(x)$ is strictly monotone, then the monotonicity in the conclusion is also strict.

Lemma 2.2 (See [21], Theorem 3.21) (1) The function $r \mapsto\left[\mathcal{E}(r)-\left(1-r^{2}\right) \mathcal{K}(r)\right] / r^{2}$ is strictly increasing from $(0,1)$ onto $(\pi / 4,1)$.

(2) The function $r \mapsto\left(1-r^{2}\right)^{\lambda} \mathcal{K}(r)$ is strictly decreasingfrom $(0,1)$ onto $(0, \pi / 2)$ if $\lambda \geq 1 / 4$.

Lemma 2.3 The function $r \mapsto\left[2\left(1-r^{2}\right) \mathcal{E}(r)-\pi\right] / r^{2}$ is strictly increasing from $(0,1)$ onto $(-5 \pi / 4,-\pi)$.

Proof Let $f_{1}(r)=2\left(1-r^{2}\right) \mathcal{E}(r)-\pi, f_{2}(r)=r^{2}$ and $f(r)=\left[2\left(1-r^{2}\right) \mathcal{E}(r)-\pi\right] / r^{2}$. Then

$$
\begin{aligned}
& f_{1}\left(0^{+}\right)=f_{2}(0)=0, \quad f(r)=\frac{f_{1}(r)}{f_{2}(r)}, \\
& f\left(1^{-}\right)=-\pi
\end{aligned}
$$

and simple computations lead to

$$
\frac{f_{1}^{\prime}(r)}{f_{2}^{\prime}(r)}=-3 \mathcal{E}(r)+\frac{\mathcal{E}(r)-\left(1-r^{2}\right) \mathcal{K}(r)}{r^{2}} .
$$

It follows from Lemma 2.2(1), (2.2), and (2.4) that $f_{1}^{\prime}(r) / f_{2}^{\prime}(r)$ is strictly increasing on $(0,1)$ and

$$
f\left(0^{+}\right)=\lim _{r \rightarrow 0^{+}} \frac{f_{1}^{\prime}(r)}{f_{2}^{\prime}(r)}=-\frac{5 \pi}{4} .
$$

Therefore, Lemma 2.3 follows from Lemma 2.1, (2.2), (2.3), (2.5), and the monotonicity of $f_{1}^{\prime}(r) / f_{2}^{\prime}(r)$.

Lemma 2.4 Let $p \in(0,1), r \in(0,1)$ and

$$
f(r)=\frac{4\left[\mathcal{E}(r)-\left(1-r^{2}\right) \mathcal{K}(r)\right]}{r^{2}}+\frac{2\left(1-r^{2}\right) \mathcal{E}(r)-\pi}{r^{2}}+\pi(1-p) .
$$

Then the following statements are true:

(1) If $p=3 / 4$, then $f(r)>0$ for all $r \in(0,1)$;

(2) If $p=4(4-\pi) /[\pi(\pi-2)]=0.9573 \cdots$, then there exists $r_{0} \in(0,1)$ such that $f(r)<0$ for $r \in\left(0, r_{0}\right)$ and $f(r)>0$ for $r \in\left(r_{0}, 1\right)$. 
Proof For part (1), if $p=3 / 4$, then (2.6) becomes

$$
f(r)=\frac{4\left[\mathcal{E}(r)-\left(1-r^{2}\right) \mathcal{K}(r)\right]}{r^{2}}+\frac{2\left(1-r^{2}\right) \mathcal{E}(r)-\pi}{r^{2}}+\frac{\pi}{4},
$$

and Lemma 2.2(1) and Lemma 2.3 lead to

$$
f(r)>4 \times \frac{\pi}{4}-\frac{5 \pi}{4}+\frac{\pi}{4}=0
$$

for all $r \in(0,1)$.

For part (2), if $p=4(4-\pi) /[\pi(\pi-2)]$, then it follows from Lemma 2.2(1), Lemma 2.3, and (2.6) that

$$
\begin{aligned}
& f\left(0^{+}\right)=-\frac{64-3 \pi^{2}-10 \pi}{4(\pi-2)}=-0.6515 \cdots<0, \\
& f\left(1^{-}\right)=\frac{8(\pi-3)}{\pi-2}=0.9922 \cdots>0
\end{aligned}
$$

and $f(r)$ is strictly increasing on $(0,1)$.

Therefore, part (2) follows from (2.7) and (2.8) together with the monotonicity of $f(r)$.

\section{Main results}

Theorem 3.1 The double inequality

$$
\alpha N_{A Q}(a, b)+(1-\alpha) A(a, b)<T^{*}(a, b)<\beta N_{A Q}(a, b)+(1-\beta) A(a, b)
$$

holds for all $a, b>0$ with $a \neq b$ if and only if $\alpha \leq 3 / 4$ and $\beta \geq 4(4-\pi) /[\pi(\pi-2)]=$ $0.9573 \cdots$.

Proof Since $A(a, b), T^{*}(a, b)$ and $N_{A Q}(a, b)$ are symmetric and homogeneous of degree 1, without loss of generality, we assume that $a>b$. Let $r=(a-b) /(a+b) \in(0,1)$ and $p \in(0,1)$. Then (2.1) leads to

$$
T^{*}(a, b)=\frac{2}{\pi} A(a, b)\left[2 \mathcal{E}(r)-\left(1-r^{2}\right) \mathcal{K}(r)\right]
$$

It follows from (1.4), Lemma 2.2(2), and (3.2) that

$$
\begin{aligned}
& \frac{T^{*}(a, b)-A(a, b)}{N_{A Q}(a, b)-A(a, b)}=\frac{\frac{2}{\pi}\left[2 \mathcal{E}(r)-\left(1-r^{2}\right) \mathcal{K}(r)\right]-1}{\frac{\left(1+r^{2}\right) \arctan (r)}{2 r}-\frac{1}{2}}, \\
& T^{*}(a, b)-\left[p N_{A Q}(a, b)+(1-p) A(a, b)\right]=\frac{p\left(1+r^{2}\right)}{2 r} A(a, b) F(r),
\end{aligned}
$$

where

$$
\begin{aligned}
& F(r)=\frac{4 r\left[2 \mathcal{E}(r)-\left(1-r^{2}\right) \mathcal{K}(r)\right]+\pi(p-2) r}{p \pi\left(1+r^{2}\right)}-\arctan (r), \\
& F\left(0^{+}\right)=0,
\end{aligned}
$$




$$
\begin{aligned}
& F\left(1^{-}\right)=\frac{4(4-\pi)+p \pi(2-\pi)}{4 p \pi}, \\
& F^{\prime}(r)=\frac{2 r^{2}}{p \pi\left(1+r^{2}\right)^{2}} f(r),
\end{aligned}
$$

where $f(r)$ is defined as in Lemma 2.4.

We divide the proof into two cases.

Case $1 p=3 / 4$. Then Lemma 2.4(1) and (3.7) lead to the conclusion that $F(r)$ is strictly increasing on $(0,1)$. Therefore,

$$
T^{*}(a, b)>\frac{3}{4} N_{A Q}(a, b)+\frac{1}{4} A(a, b)
$$

follows from (3.4) and (3.5) together with the monotonicity of $F(r)$.

Case $2 p=4(4-\pi) /[\pi(\pi-2)]$. Then (3.6) becomes

$$
F\left(1^{-}\right)=0
$$

and Lemma 2.4(2) and (3.7) imply that there exists $r_{0} \in(0,1)$ such that $F(r)$ is strictly decreasing on $\left(0, r_{0}\right]$ and strictly increasing on $\left[r_{0}, 1\right)$. Therefore,

$$
T^{*}(a, b)<\frac{4(4-\pi)}{\pi(\pi-2)} N_{A Q}(a, b)+\left[1-\frac{4(4-\pi)}{\pi(\pi-2)}\right] A(a, b)
$$

follows from (3.4), (3.5), (3.8), and the piecewise monotonicity of $F(r)$.

Next, we prove that $\alpha=3 / 4$ and $\beta=4(4-\pi) /[\pi(\pi-2)]$ are the best possible parameters such that the double inequality (3.1) holds for all $a, b>0$ with $a \neq b$. It is not difficult to verify that

$$
\begin{aligned}
& \lim _{r \rightarrow 0^{+}} \frac{\frac{2}{\pi}\left[2 \mathcal{E}(r)-\left(1-r^{2}\right) \mathcal{K}(r)\right]-1}{\frac{\left(1+r^{2}\right) \arctan (r)}{2 r}-\frac{1}{2}}=\frac{3}{4}, \\
& \lim _{r \rightarrow 1^{-}} \frac{\frac{2}{\pi}\left[2 \mathcal{E}(r)-\left(1-r^{2}\right) \mathcal{K}(r)\right]-1}{\frac{\left(1+r^{2}\right) \arctan (r)}{2 r}-\frac{1}{2}}=\frac{4(4-\pi)}{\pi(\pi-2)} .
\end{aligned}
$$

If $\alpha>3 / 4$, then (3.3) and (3.9) imply that there exists $0<\delta_{1}<1$ such that

$$
T^{*}(a, b)<\alpha N_{A Q}(a, b)+(1-\alpha) A(a, b)
$$

for all $a>b>0$ with $(a-b) /(a+b) \in\left(0, \delta_{1}\right)$.

If $\beta<4(4-\pi) /[\pi(\pi-2)]$, then (3.3) and (3.10) imply that there exists $0<\delta_{2}<1$ such that

$$
T^{*}(a, b)>\beta N_{A Q}(a, b)+(1-\beta) A(a, b)
$$

for all $a>b>0$ with $(a-b) /(a+b) \in\left(1-\delta_{2}, 1\right)$.

Theorem 3.2 Let $\lambda, \mu \in(1 / 2,1)$. Then the double inequality

$$
Q[\lambda a+(1-\lambda) b, \lambda b+(1-\lambda) a]<T^{*}(a, b)<Q[\mu a+(1-\mu) b, \mu b+(1-\mu) a]
$$


holds for all $a, b>0$ with $a \neq b$ if and only if $\lambda \leq 1 / 2+\sqrt{2} / 4=0.8535 \cdots$ and $\mu \geq 1 / 2+$ $\sqrt{16 / \pi^{2}-1} / 2=0.8940 \cdots$.

Proof Without loss of generality, we assume that $a>b>0$. Let $r=(a-b) /(a+b) \in(0,1)$ and $p \in(0,1)$. Then from (3.2) and

$$
Q[p a+(1-p) b, p b+(1-p) a]=A(a, b) \sqrt{(2 p-1)^{2} r^{2}+1}
$$

we get

$$
\begin{aligned}
T^{*} & (a, b)-Q[p a+(1-p) b, p b+(1-p) a] \\
& =\left[\frac{2}{\pi}\left(2 \mathcal{E}(r)-\left(1-r^{2}\right) \mathcal{K}(r)\right)-\sqrt{(2 p-1)^{2} r^{2}+1}\right] A(a, b) \\
& =\frac{g(r)}{\frac{2}{\pi}\left(2 \mathcal{E}(r)-\left(1-r^{2}\right) \mathcal{K}(r)\right)+\sqrt{(2 p-1)^{2} r^{2}+1}} A(a, b),
\end{aligned}
$$

where

$$
\begin{aligned}
& g(r)=\frac{4}{\pi^{2}}\left[2 \mathcal{E}(r)-\left(1-r^{2}\right) \mathcal{K}(r)\right]^{2}-(2 p-1)^{2} r^{2}-1, \\
& g\left(0^{+}\right)=0, \\
& g\left(1^{-}\right)=\frac{16}{\pi^{2}}-(2 p-1)^{2}-1 .
\end{aligned}
$$

Let

$$
g_{1}(r)=g^{\prime}(r) / r
$$

Then (3.13) and Lemma 2.2 lead to

$$
\begin{aligned}
& g_{1}(r)=\frac{8}{\pi^{2}}\left[2 \mathcal{E}(r)-\left(1-r^{2}\right) \mathcal{K}(r)\right] \frac{\mathcal{E}(r)-\left(1-r^{2}\right) \mathcal{K}(r)}{r^{2}}-2(2 p-1)^{2}, \\
& g_{1}\left(0^{+}\right)=1-2(2 p-1)^{2}, \\
& g_{1}\left(1^{-}\right)=\frac{16}{\pi^{2}}-2(2 p-1)^{2} .
\end{aligned}
$$

We divide the proof into two cases.

Case $1 p=1 / 2+\sqrt{2} / 4$. Then (3.18) becomes

$$
g_{1}\left(0^{+}\right)=0 .
$$

From Lemma 2.2(1) and $d\left[2 \mathcal{E}(r)-\left(1-r^{2}\right) \mathcal{K}(r)\right] / d r=\left[\mathcal{E}(r)-\left(1-r^{2}\right) \mathcal{K}(r)\right] / r$ we know that the function $r \mapsto 2 \mathcal{E}(r)-\left(1-r^{2}\right) \mathcal{K}(r)$ is strictly increasing on $(0,1)$. Then from Lemma 2.2(1) and (3.17) together with (3.20) we know that $g_{1}(r)$ is strictly increasing on $(0,1)$ and

$$
g_{1}(r)>g_{1}\left(0^{+}\right)=0
$$


for $r \in(0,1)$. Therefore,

$$
T^{*}(a, b)>Q\left[\left(\frac{1}{2}+\frac{\sqrt{2}}{4}\right) a+\left(\frac{1}{2}-\frac{\sqrt{2}}{4}\right) b,\left(\frac{1}{2}+\frac{\sqrt{2}}{4}\right) b+\left(\frac{1}{2}-\frac{\sqrt{2}}{4}\right) a\right]
$$

follows from (3.12), (3.14), (3.16), and (3.21).

Case $2 p=1 / 2+\sqrt{16 / \pi^{2}-1} / 2$. Then (3.15), (3.18), and (3.19) lead to

$$
\begin{aligned}
& g\left(1^{-}\right)=0 \\
& g_{1}\left(0^{+}\right)=-\frac{32-3 \pi^{2}}{\pi^{2}}<0, \\
& g_{1}\left(1^{-}\right)=\frac{2 \pi^{2}-16}{\pi^{2}}>0 .
\end{aligned}
$$

It follows from (3.16), (3.23), and (3.24) together with the monotonicity of $g_{1}(r)$ that there exists $r^{*} \in(0,1)$ such that $g(r)$ is strictly decreasing on $\left(0, r^{*}\right]$ and strictly increasing on $\left[r^{*}, 1\right)$. Therefore,

$$
T^{*}(a, b)<Q[p a+(1-p) b, p b+(1-p) a]
$$

follows from (3.12), (3.14), (3.22), and the piecewise monotonicity of $g(r)$.

Next, we prove that $\lambda=1 / 2+\sqrt{2} / 4$ and $\mu=1 / 2+\sqrt{16 / \pi^{2}-1} / 2$ are the best possible parameters in $(1 / 2,1)$ such that the double inequality (3.11) holds for all $a, b>0$ with $a \neq b$. If $1 / 2+\sqrt{2} / 4<p<1$, then (3.18) leads to

$$
g_{1}\left(0^{+}\right)<0
$$

Equations (3.12), (3.14), and (3.16) and inequality (3.25) imply that there exists $\delta_{3} \in(0,1)$ such that

$$
T^{*}(a, b)<Q[p a+(1-p) b, p b+(1-p) a]
$$

for all $a>b>0$ with $(a-b) /(a+b) \in\left(0, \delta_{3}\right)$.

If $1 / 2<p<1 / 2+\sqrt{16 / \pi^{2}-1} / 2$, then (3.15) leads to

$$
g\left(1^{-}\right)>0
$$

Equation (3.12) and inequality (3.26) imply that there exists $\delta_{4} \in(0,1)$ such that

$$
T^{*}(a, b)>Q[p a+(1-p) b, p b+(1-p) a]
$$

for all $a>b>0$ with $(a-b) /(a+b) \in\left(1-\delta_{4}, 1\right)$.

Let $r \in(0,1), r^{*}=r^{2} /\left(1+\sqrt{1-r^{2}}\right)^{2}, a=1, b=\sqrt{1-r^{2}}, \alpha=3 / 4, \beta=4(4-\pi) /[\pi(\pi-2)], \lambda=$ $1 / 2+\sqrt{2} / 4$, and $\mu=1 / 2+\sqrt{16 / \pi^{2}-1} / 2$. Then Theorems 3.1 and 3.2 lead to Corollary 3.3 as follows. 
Corollary 3.3 The double inequalities

$$
\begin{aligned}
& \frac{\pi\left(1+\sqrt{1-r^{2}}\right)}{32}\left[5+3\left(r^{*}+\frac{1}{r^{*}}\right) \arctan \left(r^{*}\right)\right] \\
& <\mathcal{E}(r)<\frac{1+\sqrt{1-r^{2}}}{4(\pi-2)}\left[\pi^{2}-8+2(4-\pi)\left(r^{*}+\frac{1}{r^{*}}\right) \arctan \left(r^{*}\right)\right]
\end{aligned}
$$

and

$$
\frac{\pi \sqrt{6+2 \sqrt{1-r^{2}}-3 r^{2}}}{4 \sqrt{2}}<\mathcal{E}(r)<\frac{\sqrt{8+\left(\pi^{2}-8\right) \sqrt{1-r^{2}}-4 r^{2}}}{2}
$$

hold for all $r \in(0,1)$.

\section{Competing interests}

The authors declare that they have no competing interests.

\section{Authors' contributions}

All authors contributed equally to the writing of this paper. All authors read and approved the final manuscript.

\section{Author details}

${ }^{1}$ School of Mathematics and Computation Sciences, Hunan City University, Yiyang, 413000, China. ${ }^{2}$ School of Distance Education, Huzhou Broadcast and TV University, Huzhou, 313000, China.

\section{Acknowledgements}

The authors wish to thank the anonymous referees for their careful reading of the manuscript and their fruitful comments and suggestions. The research was supported by the Major Project Foundation of the Department of Education of Hunan Province under Grant 12A026.

Received: 7 May 2015 Accepted: 27 August 2015 Published online: 17 September 2015

\section{References}

1. Toader, G: Some mean values related to the arithmetic-geometric mean. J. Math. Anal. Appl. 218(2), 358-368 (1998)

2. Neuman, E: Bounds for symmetric elliptic integrals. J. Approx. Theory 122(2), 249-259 (2003)

3. Kazi, H, Neuman, E: Inequalities and bounds for elliptic integrals.J. Approx. Theory 146(2), 212-226 (2007)

4. Kazi, H, Neuman, E: Inequalities and bounds for elliptic integrals II. In: Special Functions and Orthogonal Polynomials. Contemp. Math., vol. 471, pp. 127-138. Am. Math. Soc., Providence (2008)

5. Chu, Y-M, Wang, M-K, Qiu, S-L: Optimal combinations bounds of root-square and arithmetic means for Toader mean. Proc. Indian Acad. Sci. Math. Sci. 122(1), 41-51 (2012)

6. Chu, Y-M, Wang, M-K: Inequalities between arithmetic-geometric, Gini, and Toader means. Abstr. Appl. Anal. 2012 Article ID 830585 (2012)

7. Chu, Y-M, Wang, M-K: Optimal Lehmer mean bounds for Toader mean. Results Math. 61(3-4), 223-229 (2012)

8. Chu, Y-M, Wang, M-K, Ma, X-Y: Sharp bounds for Toader mean in terms of contraharmonic mean with applications. J. Math. Inequal. 7(1), 161-166 (2013)

9. Song, Y-Q, Jiang, W-D, Chu, Y-M, Yan, D-D: Optimal bounds for Toader mean in terms of arithmetic and contraharmonic means. J. Math. Inequal. 7(4), 751-757 (2013)

10. Li, W-H, Zheng, M-M: Some inequalities for bounding Toader mean. J. Funct. Spaces Appl. 2013, Article ID 394194 (2013)

11. Yun, H, Qi, F: A double inequality for bounding Toader mean by the centroidal mean. Proc. Indian Acad. Sci. Math. Sci. 124(4), 527-531 (2014)

12. Yun, $\mathrm{H}, \mathrm{Qi}$, F: The best bounds for Toader mean in terms of the centroidal and arithmetic means. arXiv:1303.2451v1 [math. CA]

13. Vuorinen, M: Hypergeometric functions in geometric function theory. In: Special Functions and Differential Equations (Madras, 1977), pp. 119-126. Allied Publishers, New Delhi (1998)

14. Qiu, S-L, Shen, J-M: On two problems concerning means. J. Hangzhou Inst. Electr. Eng. 17(3), 1-7 (1997) (in Chinese)

15. Barnard, RW, Pearce, K, Richards, KC: An inequality involving the generalized hypergeometric function and the arc length of an ellipse. SIAM J. Math. Anal. 31(3), 693-699 (2000)

16. Alzer, H, Qiu, S-L: Monotonicity theorems and inequalities for the complete elliptic integrals. J. Comput. Appl. Math. 172(2), 289-312 (2004)

17. Chu, Y-M, Wang, M-K, Qiu, S-L, Qiu, Y-F: Sharp generalized Seiffert mean bounds for Toader mean. Abstr. Appl. Anal. 2011, Article ID 605259 (2011)

18. Neuman, E, Sándor, J: On the Schwab-Borchardt mean. Math. Pannon. 14(2), 253-266 (2003)

19. Neuman, E, Sándor, J: On the Schwab-Borchardt mean II. Math. Pannon. 17(1), 49-59 (2006)

20. Neuman, E: On a new bivariate mean. Aequ. Math. 88(3), 277-289 (2014)

21. Anderson, GD, Vamanamurthy, MK, Vuorinen, M: Conformal Invariants, Inequalities, and Quasiconformal Maps. Wiley, New York (1997) 